\title{
Volume Cardiac SPECT Image Registration
}

\author{
Santiago Murillo, Isabel Navazo, Alvar Vinacua \\ Universitat Politècnica de Catalunya \\ \{smurillo, isabel, alvar \}@1si.upc.edu
}

\begin{abstract}
The registration of cardiac images is relevant for the diagnosis of cardiac pathologies. In the case of SPECT (Single Photon Emission Computer Tomography) datasets, this is difficult because of the low resolution of the images. In this paper we present a new registration method for $3 \mathrm{D}$ images with poor resolution. Our method is based on the maximization of the mutual information (MI) using a new interpolation method which significantly improves the result of an MI-based strategy on low resolution images. We also use a different optimization strategy than other MI-based algorithms, typically achieving errors of the order of one pixel. We also discuss experimental results of our method.
\end{abstract}

\section{Introduction}

The high rates of cardiac disease in our society require the development of effective and fast techniques of diagnosis which allow the timely beginning of an adequate treatment. The acquisition of cardiac images through nuclear medicine techniques such as SPECT (Single Photon Emission Computed Tomographic), see figure 1, captures the blood perfusion in the myocardium.

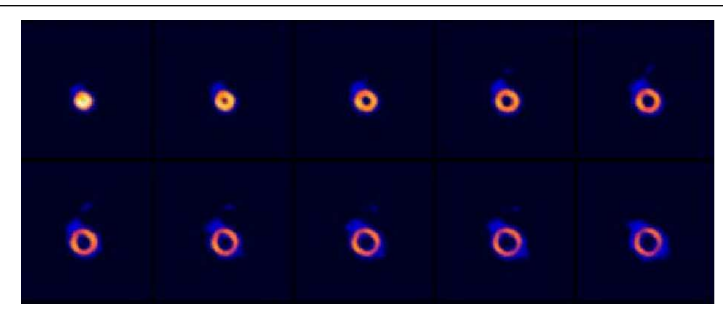

Figure 1. Cardiac SPECT series.

An infarction reduces the blood irrigating portions of the heart. To establish the extent of damage and the potential for success with treatment, SPECT images are effec- tive. The diagnosis is performed by the cardiac specialist who visually examines the set of images from the patient's rest and stress SPECT. Areas with small perfusion under stress but correctly irrigated at rest are of interest, as they may be healed under adequate treatment. Also of interest is the comparative analysis of the blood perfusion in the myocardium of the patient over time.

In this article we present an automatic technique of registration of SPECT images (SPECT-rest and SPECT-stress, for example). The algorithm is based on the use of the information theory as introduced by A.Collignon in [2], in order to align the data of both images. Unlike previous works, our algorithm deals with a three-dimensional model directly (the set of all slices in a SPECT image). The value of a voxel of each image measures the blood perfusion in a particular zone of the myocardium. The algorithm finds the geometric transformation for aligning one of the volumetric image, which is labelled as floating image, with the other volumetric image, which is labelled as reference image. The results obtained show the accuracy of the our method for the registration of SPECT images. We are beginning the phase of clinical testing.

Section 2 presents briefly the state of the art of SPECT image registration. Next in section 3 we shortly present the theory of information used in image registration. In section 4 we present our method, and finally in section 5 we present the experimental results obtained with our method.

The main contributions of this work are:

1. Cardiac SPECT image registration with improved accuracy.

2. We provide a tool for the quantitative and qualitative analysis of the variation of blood perfusion by the simultaneous and registered visualization of both volumetric images.

3. We deal with the low resolution and the noise of the SPECT images, obtaining subvoxel accuracy.

4. We propose a new method for the calculation of the interpolation weights which are used to calculate the mutual information (IM) in each iteration. 


\section{Previous Work}

The SPECT image registration is a developing research field. Its main difficulty is that the SPECT data are usually gathered at an image resolution of $64 \times 64$ pixels compared with $512 \times 512$ pixels of the $\mathrm{CT}$ and MRI images. Additionally, the SPECT images have a high level of noise.

We have found a large number of references about medical image registration, most of which are applied to MRI, CT and Ultrasound images. The more relevant approaches are included in the surveys of A. Maintz in [5] , T.Mäkela et al in [7] and J. Pluim in [10].

In relation to cardiac SPECT images Z.He presents in [3] a method based on the manual selection of feature points on certain layers of the image, the accuracy of this method depends of the skills of the cardiac specialist.

To the best of our knowledge no previously published algorithm is able to automatically align SPECT image and deals with the whole volumetric model at once.

Some researchers working in the medical image registration field, have used the Mutual Information (MI) as a similarity measure and they have found that this measure is statistically robust. Some of those approaches were presented by: F. Maes et al in [4], H.Chen et al [1], A.Collignon [2], C. Mayer et al [6], J. Pluim [9, 8], J.Rigau et al [11] and P. Viola et al [12]. All of them compute the images alignment by iteratively optimizing the MI between the reference 2D image and transformed 2D image. Collignon [2] and J. Pluim [8] use the Partial Volumetric Interpolation (PVI) with CT and MRI images, in order to reduce the interpolation artifacts.

Our method has the same basis as the previous algorithms for image registration using MI, but we have adapted them to the characteristics of SPECT images, since the simple application of the previous algorithms is not effective, in this case, due to the smaller resolution and higher noise of SPECT images. For these reasons, the previous algorithms do not produce satisfactory results (see section 5). One of the contributions of this work is a more precise interpolation technique which is explained in detail in section 4 .

\section{Information Theory applied to image regis- tration}

Below we present a brief review of the theoretical framework used in the description of our method.

\subsection{Probability Distribution Estimation:}

Considering the intensity values from the images ( $A$ and $B$ ) like a set of samples of a random variable, it is possi- ble to estimate the marginal distributions $(P(A)$ and $P(B))$ from the relative frequency of each intensity value ( $a$ and $b$ ). In the same way, the joint distribution $(P(A, B))$ could be estimated from the relative frequency of coincidences of the pair of values $(a, b)$, tallied in a joint histogram $(h(A, B))$. Each entry in a joint histogram contains the number of voxels in the image that contain a particular combination of feature values ( $a$ and $b$ ). The marginal relative frequency can be estimated by the addition by rows and columns respectively of the normalized joint histogram, that is:

$$
\begin{gathered}
p(a, b)=\frac{h(a, b)}{\sum_{a, b} h(a, b)} \\
p(a)=\sum_{b} p(a, b), \quad p(b)=\sum_{a} p(a, b)
\end{gathered}
$$

\subsection{Mutual Information (MI)}

In the field of image registration, the MI — denoted by $I(A, B)$ - is considered as the measure of information that has the floating image $A$ related to the reference image $B$. In other words, it is possible to consider the MI as a measure of the uncertainty of the image $A$ with respect to the image $B$.

Considering the discussion in section $3.1, I(A, B)$ can be defined as:

$$
I(A, B)=\sum_{a}^{m} \sum_{b}^{n} p(a, b) \log \frac{p(a, b)}{p(a) p(b)}
$$

We seek a transformation $T$ of image $A$ that will make the mutual information maximal, i.e. Ts.t.I $(T(A), B)=$ $\max _{T^{\prime}} I\left(T^{\prime}(A), B\right)$. This means that the dependence between the two images is the maximum dependence possible.

\subsection{Classical algorithm for Image registration us- ing Mutual Information}

In this section we describe the classical MI-based registration algorithm.

First, in order to eliminate the image noise, most authors use a Gaussian kernel filter. They also perform a preregistration with the objective of accelerating the convergence of the optimization. For the optimization most of the authors use the multidirectional search algorithm of Powell. They assume that the MI is a mono-modal function in each parameter. This assumption does not hold for low resolution images, such as the SPECT images. 
Figure 2 shows the general algorithm for image registration. The interpolation method affects the calculation of the joint histogram in each iteration. To compute the mutual information as discussed above, we would need to resample the transformed image with the same discretization of the reference image, through some interpolation scheme. In the latest approaches [8], [12] the authors use the PVI in order to reduce the effect of the bilinear interpolation in the accuracy of the registration algorithm. However the application of this interpolation method to the registration of SPECT images does not produce satisfactory results. (See section 5). On the other hand, some authors use bicubic interpolation. In our case this is not applicable, as using a higher order interpolation method would amplify the artifacts of registration results, given the resolution of SPECT images.

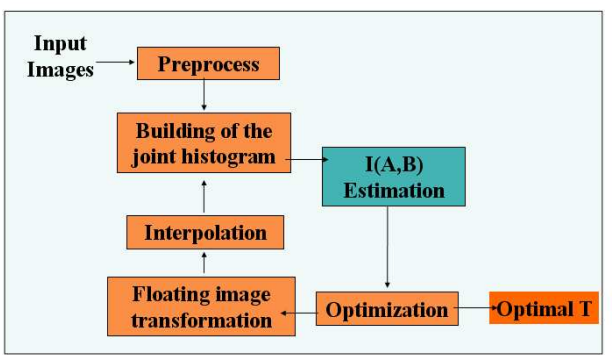

Figure 2. General Algorithm for image registration using $\mathrm{MI}$

\section{Our method for registration of cardiac vol- umetric SPECT images.}

Our method is based on the general registration algorithm seen in Figure 2, which we have modified with new techniques. These techniques improve accuracy and yield a robust algorithm. In particular, for the computation of the mutual information, we consider only the significant values that correspond to blood perfusion, we also use an optimization technique able to deal with multiple local maxima, which is therefore more suitable for cardiac SPECT registration. More important, we introduce a new interpolation method for the computation of the joint histogram of intensities between the floating image and the reference image. Below we explain each step of our method.

1. Input information and preprocess. We input all slices of two SPECT images of resolution 64 x 64 x 24 voxels. The first set of slices is labelled as reference image an the other as floating image.
We then perform the selection of significant values. In contrast with $\mathrm{CT}$ and MR images, where most of the voxels have significant information, in the cardiac SPECT images only some voxels are significant. Additionally, the presence of noise distorts the information in the voxels. In order to extract the relevant information and reduce the noise, we consider those voxels whose values are less than $35 \%$ of the maximum intensity value in each image as background. Furthermore we do not include the background in the MI calculation.

2. Pre-registration. The shape of the left ventricle is approximated by an ellipsoid (see 3D left ventricle figure in table 2). We perform the pre-registration aligning the two volumetric barycenters and next we align the long axis on both ventricles. We estimate the long axes by the axis which passes through the volumetric barycenter and the barycenter of the last slice with relevant information.

3. Optimization: we use an optimization method with truncated normal samples of the solution space. The optimization process returns the maximum mutual information that has been achieved and the corresponding transformation parameters $\alpha=[\Theta, \beta, \gamma, \Delta x, \Delta y, \Delta z]$. In section 4.1.1 we describe the MI computation and the interpolation method used.

4. Optimal transformation application and rendering In order to visualize the results in an intuitive way, we apply the optimal transformation to the floating image and then we render each one in an independent color channel, which allows us to jointly visualize the volumes and identify the mis-registration in those zones where only one of the colors used is predominant. Results in section 5 are presented in this fashion.

\subsection{Geometric Transformation and Interpolation Method}

In this section we define the rigid geometric transformation $T$ that we use in each iteration of the optimization loop. The transformation $T_{\alpha}$ with $\alpha=[\Delta x, \Delta y, \Delta Z, \Theta, \beta, \gamma]$, is the composition of a rotation through angles $\Theta, \beta, \gamma$ around the axes $X, Y, Z$ and a translation $\Delta x, \Delta y, \Delta z$. The transformed grid will not be aligned with that of the reference image (see Figure 3), so it would be necessary to compute the contributions of the different voxels in the transformed grid to the voxels it would overlap in the grid of the reference image.

Recent algorithms compute the joint histogram using Partial Volume Interpolation (PVI), shown at the left of figure 3. PVI runs faster doing a rough estimation of the 


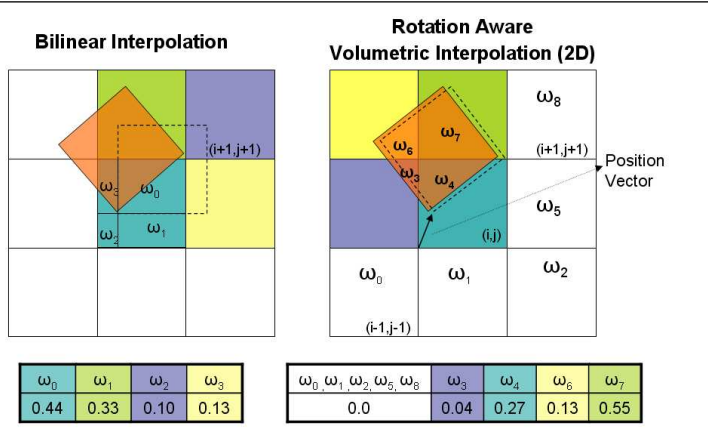

Figure 3. Interpolation Weights Estimation

weights and directly computing its effects on the joint histogram. The PVI computation is exact only if there are no rotations. In the presence of rotations it is extremely inaccurate.

4.1.1. Rotation Aware Volumetric Interpolation. Figure 3 shows a two-dimensional comparison between the calculation of PVI and our interpolation method. In PVI the weights from bilinear interpolation are used. On the left side of figure 3 , the resulting weights are the areas labeled $\omega_{0} \ldots \omega_{3}$. It is easy to see that they correspond exactly with the overlaps of the transformed voxel with the reference voxels, if there is no rotation (see dashed square). Notice that if there is a rotation, the weights may even be assigned to voxels that are not overlapped at all.

Precision can be improved by computing the exact contribution of the voxel to each overlapped voxel. However the exact computation of all these intersection volumes is far too expensive to be performed for each voxel and for each iteration of the algorithm. Instead, for each iteration we build a sufficiently dense sample set of cases, and use the closet sample to retrieve the weights for each voxel. As a result, our method, depicted on the right side of Figure 3 , would compute exact coefficients for the dashed square, which is rotated by the same angle as the figure, but slightly shifted in position from the actual voxel being considered, yielding a much improved error in this case.

More precisely, the Rotation Aware Volumetric Interpolation algorithm consists of the following steps:

1. Create two grids $M_{1}$ and $M_{2}$ with the same voxel dimensions than the SPECT images and a resolution of $10 \times 10 \times 10$. This resolution has been found to be enough for estimating the posible volumes of intersection.

2. Apply the rotation part of a test transformation $T_{\alpha}$ to $M_{2}$ getting $\operatorname{Rot}\left(T_{\alpha}\right)\left(M_{2}\right)$.
3. Estimate the intersection volumes: considering one voxel from a grid as a solid object, and considering that one voxel, when rotated, cannot be placed over a grid of voxels of the same size overlapping more than 27 voxels.

With the grids $M_{1}$ and $\operatorname{Rot}\left(T_{\alpha}\right)\left(M_{2}\right)$ superposed, it is possible to compute for each voxel in the second grid, the 27 volumes of intersection $\omega_{i}$ with voxels of $M_{1}, i=0, \ldots, 26$. Note that $\Sigma_{i=0}^{26} \omega_{i}=1$, since the volume of a voxel is equal to 1 . For each voxel processed, we store the position vector (see right side of Figure 3), and the 27 weights $\omega_{i}$.

4. Weights lookup table: during the computation of the joint histogram, we will need to search, for each voxel, an adequate sample among those computed at the previous step. We chose that sample whose position vector is closest to the actual position vector of the voxel being considered. This can require a lot of computational effort, so in order to speed up the search, we build a tridimensional lookup table of dimension $100 \times 100 \times 100$. Since each component of a position vector is in the range $[0,1]$, we scale the position vectors by 100 , and take integer parts of their coordinates to address this table (which can be seen as a simple hash technique). We store each sample computed above in this table, adding it to the list corresponding to the position indicated by its scaled position vector; for each sample we store both the position vector itself and the computed weights.

5. Computation of the Intensity joint histogram $h\left(T_{\alpha}(A), B\right)$.

For each voxel

(a) Compute the final position of the voxel in the floating image $A$ applying the test transforma$\operatorname{tion}\left(T_{\alpha}\right)$.

(b) Compute its position vector $v_{p}$ (see Figure 3 ).

(c) Select the entry in the lookup table indexed by $\left\lfloor 100 v_{p}\right\rfloor$. If there are several vectors in the same entry, we choose the closest vector to $v_{p}$.

(d) Using the 27 weights $\omega_{i}$ corresponding to the selected sample, estimate the contribution of the current voxel to the intensity joint histogram $h\left(T_{\alpha}(A), B\right)$ :

Let $b_{i}, i=0, . ., 26$ be the values of the 27 voxels in the original reference image $B$ and let $a$ be the value of intensity of the current voxel in the floating image $A$. We update the elements of the joint histogram with the corresponding weights, i.e. $h\left(a, b_{i}\right)=h\left(a, b_{i}\right)+\omega_{i}$

6. Estimate the mutual information using the new intensity joint histogram. 


\begin{tabular}{|l|l|l|l|l|}
\hline Method & $s$ & $\sigma_{t}$ & $\begin{array}{l}\text { Average accuracy for } \\
\alpha=(\Delta x, \Delta y, \Delta Z, \Theta, \beta, \gamma)\end{array}$ & Standard Deviation $\sigma_{\alpha}$ \\
\hline $\begin{array}{l}\text { Classical } \\
\text { method }\end{array}$ & 178.45 & 7.16 & $(2.58,1.45,3.41,2.48,1.32,2.16)$ & $(1.75,1.36,3.25,2.68,1.35,1.69)$ \\
\hline $\begin{array}{l}\text { Our } \\
\text { method }\end{array}$ & 235.32 & 19.42 & $(1.15,0.75,0.95,0.85,1.11,1.04)$ & $(1.12,1.41,0.85,1.02,2.25,1.42)$ \\
\hline
\end{tabular}

Table 1. Results of 60 random cardiac SPECT registration tests performed with two registration methods

Note that similarly to PVI, in our interpolation method we do not calculate new intensity values, instead we update 27 elements of the intensity joint histogram with weights which approximate the volumes of overlap between the two images.

\section{Results}

The following are the results obtained with the application of the registration algorithm to volumetric cardiac SPECT images.

Table 1 shows the results for 60 tests using random transformations to generate a second image from a real SPECT image. The second and third columns show the average registration time in seconds $(s)$ and its standard deviation $\sigma_{t}$. The fourth and fifth column show the average accuracy of the result and its standard deviation. We measure the accuracy by the absolute difference between the resulting transformation parameters and the exact solution obtained from inverting the random transformation used. The absolute differences are expressed in voxels for $\Delta x, \Delta y, \Delta z$ and expressed in degrees for $\Theta, \beta, \gamma$.

We also applied the algorithm to register a pair of StressRest SPECT images, with the result shown in row 3 of Table 2, where it can be seen that the result is adequate, in that perfused areas are properly aligned.

Figure 4 compares the result of our algorithm with the classical MI maximization algorithm using PVI

To further test the algorithm in the presence of ischemic (under-irrigated) tissue, we constructed a test example with a phantom image of a left ventricle and the same image with some voxels deleted (corresponding to a volume of $12826.16 \mathrm{~mm}^{3}$ of myocardium), and with a random transformation applied. We performed 20 tests for aligning these models, and computed the volume of the ischemic tissue. The results of the tests gave an average relative error of $1.26 \%$ (absolute error of $162.16 \mathrm{~mm}^{3}$ ) with a standard deviation of 1.25 . The maximum obtained error is $2.10 \%$ (absolute maximum error $382.05 \mathrm{~mm}^{3}$ ). The volume of each voxel is $243 \mathrm{~mm}^{3}$. These results shows a rather satisfying alignment even when the images are different.

\section{Conclusions}

We have presented an algorithm to automatically align different SPECT images of the left ventricle. Our algorithm uses a maximization of the mutual information of the images, a standard practice to align bidimensional images, but we adapt and extend these standard techniques to operate on three-dimensional images of low resolution, which the previously published approaches could not handle. The tests made with our algorithm have shown satisfactory results with consistent precision of the order of a voxel.

An important contribution of this paper is the proposed algorithm to compute the joint histogram of the images, which without sacrificing too much speed yields much improved accuracy in the presence of rotations, and especially in three dimensions.

We are presently working on the clinical validation of these results and on extending our method to deal with gated SPECT models that capture a complete cardiac cycle.

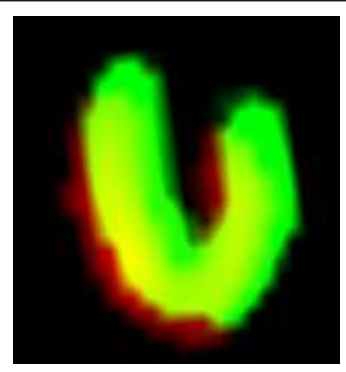

(a)

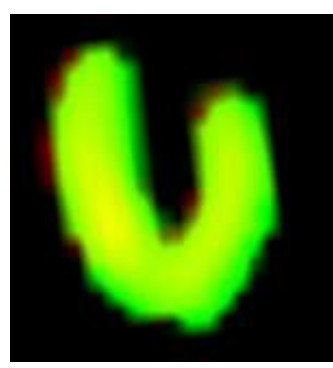

(b)
Figure 4. Comparison of the registration of two real stress-rest SPECT sagittal cuts, obtained by the classical method (a) and our method (b) 


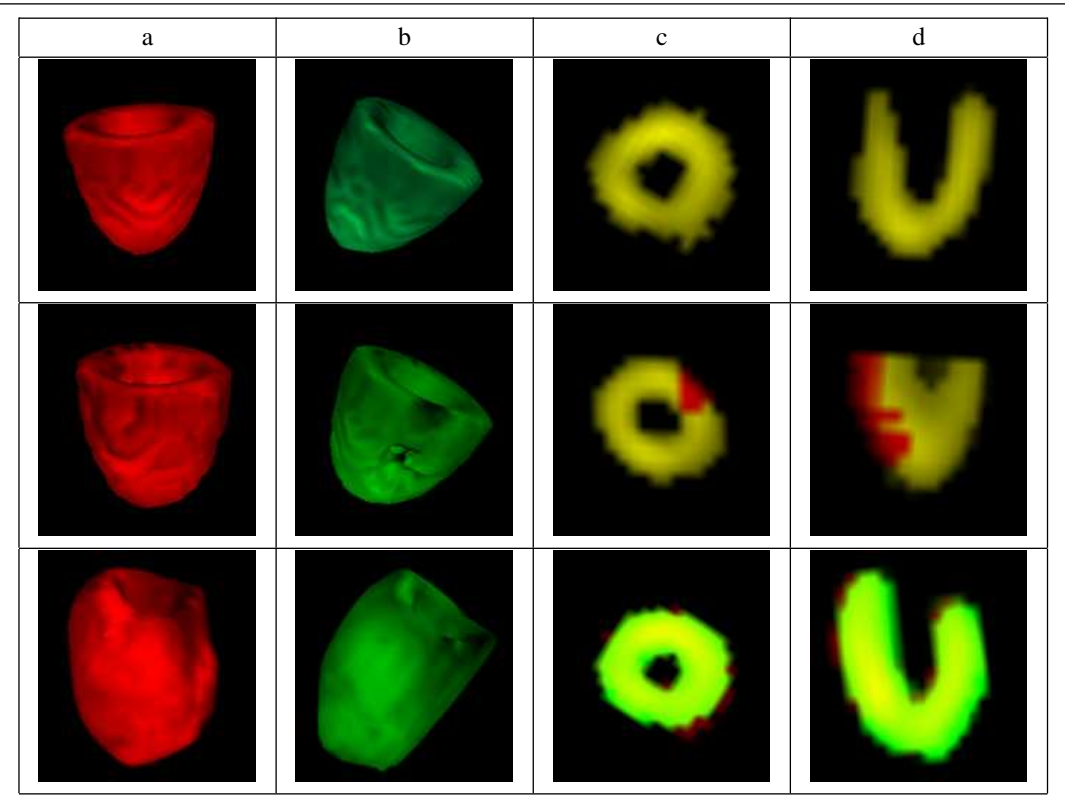

Table 2. Graphical results for registration tests.

Row 1 (test 1): Rendering of Registration of real SPECT images (red channel color) with a model generated synthetically by random transformations (green color channel ), row 2 (test 2):Registration of healthy phantom SPECT model (in red channel) and ischemic phantom model (in green color channel ), and row 3 (test 3): Registration between SPECT stress model (red channel) and SPECT rest model (green channel). Columns a) and b)Volumetric models of the left ventricle, c) one slice of the left ventricle's models overlapped, d) Sagittal cut of the overlapped models. Note that the yellow tones in c) and d) correspond to the voxels aligned with accuracy. Note that in test 3 , the "rest" image is brighter than the "stress" image, never the less the alignment achieved is satisfactory, and therefore the green channel predominates over the red channel.

\section{References}

[1] H. Chen and P. Varshney. Mutual information-based ct-mr brain image registration using generalized partial volume joint histogram estimation. IEEE Transactions on Medical Imaging, 22(9):1111-1119, 2003.

[2] A. Collignon. Multi-modality medical image registration by maximization of mutual information. $\mathrm{PhD}$ thesis, Catholic University of Leuven, Leuven, Belgium, 1998.

[3] Z. HE, J. Cauvin, and A. Veyre. Reorientation of the left ventricular long-axis on myocardial transaxial tomograms by a linear fitting method. Journal of Nuclear Medicin, 32:17941800, 1991

[4] F. Maes, A. Collignon, D. Vandermeulen, G. Marchal, and P. Suetens. Multimodality image registration by maximization of mutual information. IEEE Transactions on Medical Imaging, 16(2), 1997.

[5] A. Maintz. A survey of medical image registration. Oxford University Press, 1997.

[6] C. Meyer, J. Boes, B. Kim, and P. Bland. Evaluation of control point selection in automatic mutual information driven
3D warping. in Proc of MICCAI'98, Cambridge, USA., (1496):944-951, 1998

[7] T. Mäkela, P. Clarysse, O. Siplila, N. Pauna, Q. Pham, T. Katila, and I. Magnin. A review of cardiac image registration methods. IEEE Transactions on Medical Imaging, 21(9):1011-1021, 2002.

[8] J. Pluim. Mutual Information Based Registration of Medical Images. PhD thesis, Image Since Institut, Uthrecht University, Netherlands, 2000

[9] J. Pluim, J. Maintz, and M. Viergever. Mutual information matching and interpolation artifacts. Image Processing, 3661,1999

[10] J. Pluim, J. Maintz, and M. Viergever. Mutual information based registration of medical images: a survey. IEEE Transactions on Medical Imaging, 22(8):986-1013, 2003.

[11] J. Rigau, M.Feixas, M.Sbert, A.Bardera, and I.Boada. Medical image segmentation based on mutual information maximization. MICCAI, pages 135-142, 2004.

[12] P. Viola and W. Wells. Alignment by maximization of mutual information. International Jurnal of computer vision, 24:137-154, 1997. 